\title{
Recursos florales usados por dos especies de Bombus en un fragmento de bosque subandino (Pamplonita-Colombia)
}

\author{
Floral resources use by two species of Bombus in a subandean forest \\ fragment (Pamplonita-Colombia)
}

\author{
Mercado-G, Jorge ${ }^{1 *}$ M.Sc, Solano-R, Cristian² Biol, Wolfgang R, Hoffmann³ Biol.
}

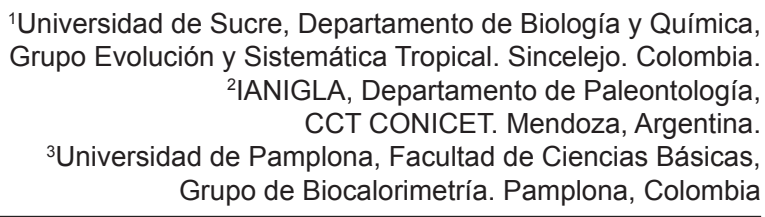

\section{Keywords:}

Pollen;

palinology;

diet;

Norte de Santander;

Hymenoptera, feces.

\section{Abstract}

It is present the results of a palynological analysis of two species of the genus Bombus in vicinity of Pamplonita-Norte of Santander. On feces samples were identified and counted (april and may of 2010) 1585 grains of pollen, within which Solanaceae, Asteraceae, Malvaceae and Fabaceae were the more abundat taxa. With regard to the diversity, April was the most rich, diverse and dominant month; however in B. pullatus we observed greater wealth and dominance. Finally, it was possible to determine that a large percentage of pollen grains that were identified as Solanaceae correspond to Solanum quitoense. The results of this study make clear the importance of palynology at the time of establishing the diet of these species, in addition to reflect its role of those species as possible provider in ecosistemic services either in wild plant as a crops in Solanaceae case.

\section{Palabras Clave:}

Polen;

palinología;

dieta;

Norte de Santander;

Hymenoptera, heces.

INFORMACIÓN

Recibido: 14-07-2016;

Aceptado: 06-11-2016.

* Correspondencia autor:

jorge.mercado@unisucre.edu.co

\section{Resumen}

Se presentan los resultados de un análisis palinológico en dos especies del género Bombus en cercanías de Pamplonita (Norte de Santander-Colombia) durante los meses de abril y mayo de 2010. A partir de muestras coprológicas se identificaron y contaron un total de 1585 granos, dentro de los cuales Solanaceae, Asteraceae, Malvaceae y Fabaceae fueron los taxa mas abundantes. En términos de diversidad, en abril se encontraron los valores más altos con respecto a los tipos de polen para ambas especies, aunque en $B$. pullatus, se observó mayor riqueza y dominancia para ambos meses. Finalmente, se puedo determinar un gran porcentaje de los granos de polen que se identificación en Solanaceae corresponden a Solanum quitoense. Los resultados presentados en este estudio dejan claro la importancia de la palinología al momento de establecer la dieta de estos organismos, además de reflejar el papel de estas especies como posible prestador de servicios ecosistemicos tanto en plantas silvestres como cultivadas en el caso de Solanum. 


\section{Introducción}

Las interacciones entre flores y animales forman parte de las relaciones ecológicas mutualistas que han sido moldeadas por la dinámica evolutiva entre los linajes de plantas y animales, produciendo diversos arreglos morfológicos, fisiológicos y de comportamiento en ambos grupos (SCHIESTL y JOHNSON, 2013). Esta relación ha afectado el sistema reproductivo en plantas y alimenticio en insectos, a tal punto que la dieta de especies del orden Hymenoptera, por ejemplo, está centrada principalmente en néctar y polen, con preferencia por plantas con ciertas cantidades de azúcares en el néctar, proteínas del polen y metabolitos secundarios como flavonoides, terpenos alcaloides y taninos que resultan ser compuestos atrayentes e importantes en su ciclo de vida (GOULSON, 2010 a; b).

Como un mecanismo para analizar la dieta de insectos como abejas y abejorros, se ha implementado el análisis palinológico en cargas de polen y mieles. Teniendo en cuenta que los granos presentan patrones morfológicos particulares para cada linaje de plantas (MERCADO-GÓMEZ et al., 2011). En este sentido este tipo de estudios han generado evidencia de la flora polinizada por diferentes especies de la familia Apidae (BARTH, 2013; ESTAY et al., 2001). En este sentido, en el Neotropico sobre Apis mellifera L. se han registrado varios trabajos palinológicos que han determinado los recursos florales y análisis detallados de la concentración de polen, debido a su importancia en la producción de miel, propóleos y polen comercial (CASTELLANOS-POTENCIANO et al., 2012; RAMÍREZ-ARRIAGA et al., 2016). Empero, sobre otros grupos como los abejorros, son poco los estudios que han analizado sus dietas a través de la palinología. Algunas aproximaciones realizadas en linajes de abejorros han ayudado a establecer el rol de Bombus en servicios ecosistemicos (KREMEN et al., 2004) y su importancia sistemas productivos agrícolas (NATES-PARRA et al., 2013; OBREGÓN et al., 2013).

Los abejorros del género Bombus sp. hacen parte del orden Hymenoptera, la tribu Bombini y la familia Apidae (GOULSON, 2010b). Sus especies presentan comportamiento que ha sido dividido en varias fases (solitaria, subsocial y social), dentro de las cuales la fases subsocial y social ocurren durante el periodo de reproducción donde las hembras posterior a la fecundación crean nidos para ovipositar, alimentar y criar a sus larvas (HOFFMANN et al., 2004).

En este sentido, para analizar la dieta de estos grupos pueden ser utilizadas dos metodológicas, la primera consiste en el estudio de las cargas polínicas (RAMÍREZ-ARRIAGA et al., 2016) y la segunda en analizar las heces directamente de los nidos
(TELLERIA, 1998; TEPER, 2006). El primer método permite conocer los recursos florales, aunque no es posible establecer sí la carga polínica será utilizada por las hembras para acumular alimentos e invernar o alimentar sus crías (GOULSON, 2010b). En el segundo caso, analizar las heces si permite realizar una aproximación más acertada sobre la dieta, teniendo en cuenta que estos son desechos del tracto digestivo (GOULSON, 2010b); además analizar la dieta en fase social (TELLERIA, 1998; TEPER, 2006).

En Colombia, sobre la cordillera oriental a las cercanías de Pamplonita (Norte de Santander), se han identificado varias especies Bombus (VEGA-SUÁREZ, 2009) de las cuales se desconoce los recursos florales utilizados como parte de su dieta en los bosques Andinos durante los periodos de nidación. Teniendo en cuenta lo anterior en la presente estudio se presentan los resultados preliminares sobre la dieta floral de Bombus pullatus (Franklin) y Bombus atratus (Franklin) durante los periodos de nidificación mediante la recolección e identificación de polen a partir de heces obtenidas en nidos, en un fragmento de bosque Subandino.

\section{Materiales y métodos}

Para analizar la dieta de $B$. pullatus y $B$. atratus se llevo a cabo inicialmente un seguimiento a varios individuos de ambas especies hasta hallar sus nidos de cría durante el mes de abril del 2010, en cercanías de Pamplonita (Norte de Santander - Colombia), sobre las coordenadas $7,45234801433981 \mathrm{~N}$ y $-72,6364992465825 \mathrm{~W}$, a una altitud de $1624 \mathrm{msnm}$. De estos nidos (uno para cada especie) y sin ocasionar daños a su estructura, se realizo una perforación desde la superficie hasta alcanzar el nido. Una vez fueron observadas larvas se obtuvieron $1 \mathrm{~cm}^{3}$ de excremento a través de una espátula, estas fueron almacenadas en recipientes plásticos con ácido acético al 10\% (evitar contaminación) debidamente rotulados. Las muestras se recolectaron con un intervalo de 30 días, resultando en: una en abril y la siguiente en mayo. No obstante, al analizar las muestras del mes de junio se observó una reducción significativa de los granos de polen ( $B$. pullatus y $B$. atratus 30 a 50 granos respectivamente) y finalmente en el mes de junio los nidos estaban abandonados y uno fue destruido.

Obtenidas las muestras, estas fueron trasladadas a la palinoteca de la Universidad de Pamplona, donde fueron acetolizadas según la metodología de ERDTMAN (1952); una vez montadas las placas en gelatina glicerinada (MERCADO-GÓMEZ, 2007), se identificaron los tipos polínicos utilizando un microscopio marca Olympus en 100x, por medio de los catálogos de MERCADO-GÓMEZ et al. (2011), BOGOTÁ (2002); 
BOGOTÁ et al. (1996), ROUBICK y MORENO (1991), entre otros, siguiendo la nomenclatura de PUNT et al. (2007).

Posterior a la identificación, se realizó el conteo de granos por medio de recorridos y observaciones aleatorias en cada placa; un mínimo 300 granos fueron contados en objetivo de 40x. Con base en los conteos e identificaciones se graficaron: número de granos asociados a géneros y familias; además de los tipos polínicos por mes en las dos especies de Bombus. Asimismo, se realizaron estimaciones de la riqueza, diversidad y dominancia con base en índices ecológicos, para determinar si existían variaciones en los meses con respecto a la estructura ecológica (PAST 3.1).

\section{Resultados y discusión}

Un total de 1585 granos de polen se contaron en las 4 muestras analizadas, dentro de los cuales se identificaron 54 palinomorfos con afinidad a 34 géneros y 13 familias que corresponden a Angiospermas. Dentro de estos clados Solanaceae, Asteraceae y Fabaceae presentaron el mayor número de granos por muestra analizada. A nivel de géneros Borreria, Cestrum, Clibadium, Phyllantus, Solanum y Taraxacum se consideraron como los más representativos (Figura 1A).

Con respecto a la concentración de polen por meses y especies, en $B$. pullatus se promedio el mayor número de granos con 451 en ambos meses; en comparación con $B$. antratus, en el cual se observaron 380 granos en promedio, aunque con diferencias significativas en
A

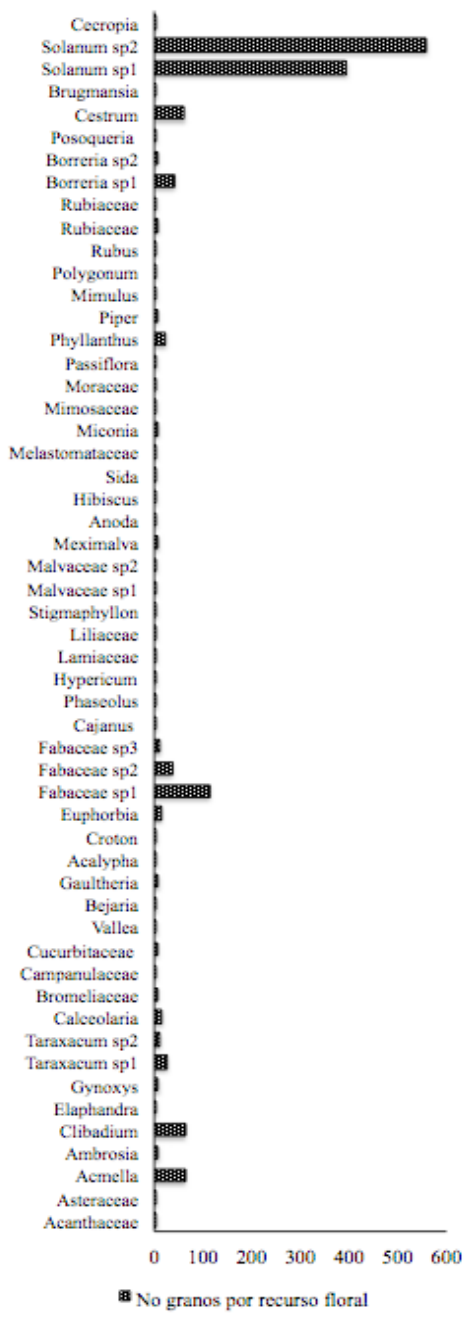

B

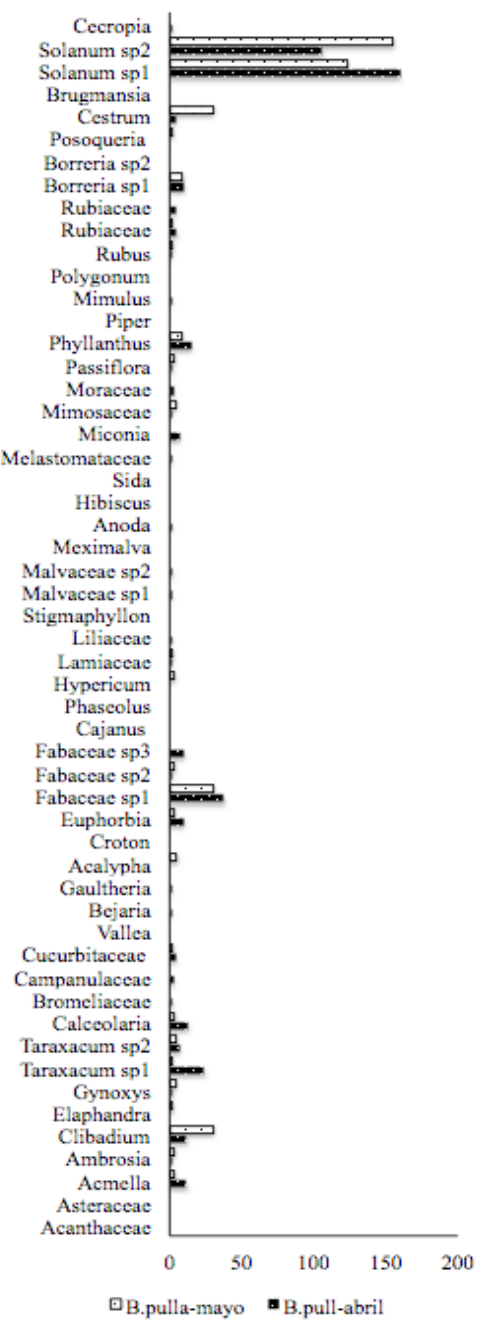

C

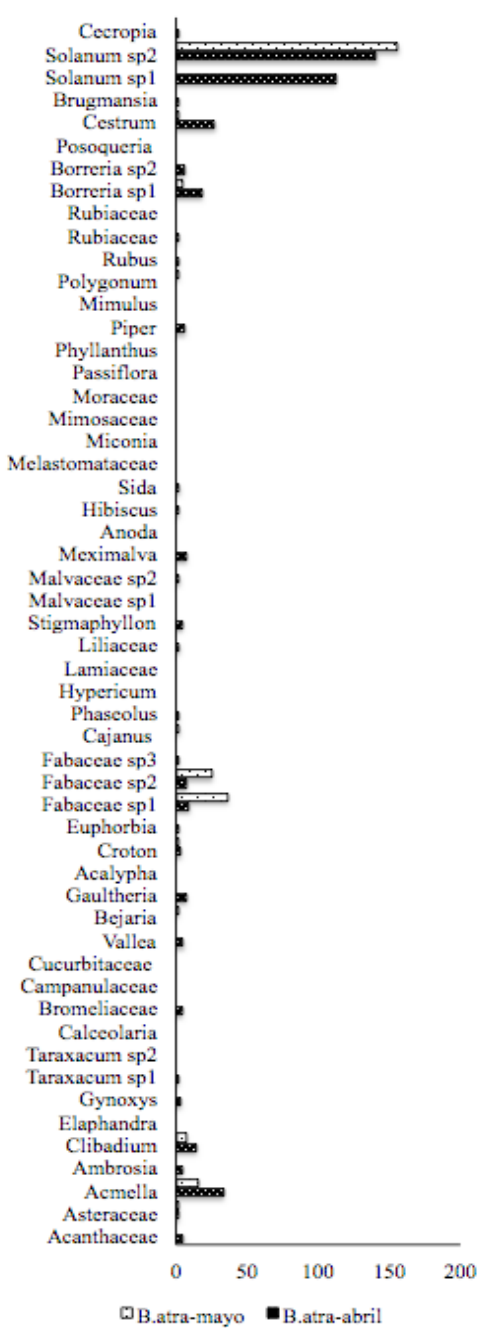

Figura 1. A, Relación entre el total de tipos polínicos identificados y la frecuencia polínica. B, relación entre el total de tipos polínicos identificados en Bombus atratus en los meses de muestreo. C, relación entre el total de tipos polínicos identificados en Bombus pullatus en los meses de muestreo. 
ambos meses. En abril por ejemplo, se contaron 431 granos, mientras que en mayo solo 250. Asimismo, notables variaciones se destacan en el número de taxones y la riqueza (Figura 2B, 2C). Por ejemplo, en abril para $B$. pullatus se identificaron 36 morfotipos, y $B$. atratus 32 ; mientras que en mayo solo 25 y 12 respectivamente (Figura 2B, 2C).

Estas variaciones en la concentración de polen en los meses de muestreo pude ser el producto de tres eventos. El primero evoca a las variaciones climáticas, ya que se ha observado en especies de melipona que la tasa de forrajeo disminuye durante periodos de lluvia (NATES-PARRA y RODRÍGUEZ, 2011). Sin embargo, la carencia de datos climáticos en el área de muestreo solo permite realizar hipótesis al respecto. Asimismo, una segunda explicación puede estar vinculada con la oferta de los recursos florares, los cuales pudieron estar con mayor disponibilidad en el mes de abril en comparación a mayo, lo cual es soportado por la mayor riqueza, diversidad y dominancia en ambas especies durante este mes, aunque con mayor incidencia en B. pullatus (Figura 2B, 2C, 2E) (NATES-PARRA y RODRÍGUEZ, 2011).
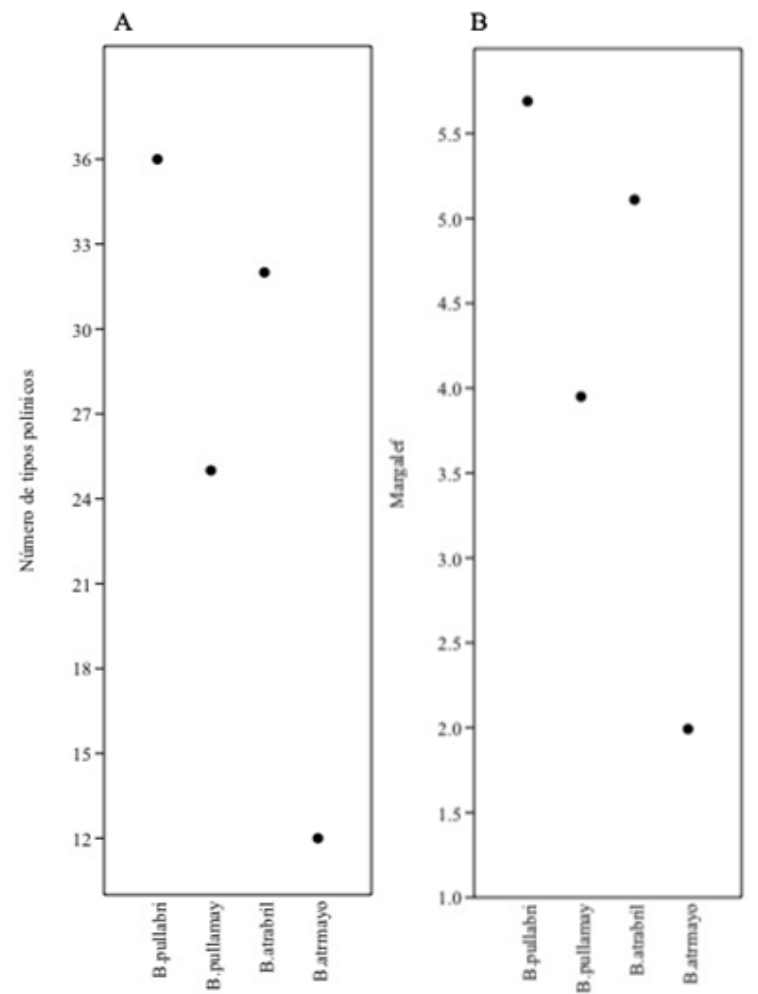

Finalmente, la ultima explicación puede estar asociada con el ciclo de vida de Bombus. En estas especies, posterior a la fecundación las hembras son fecundadas crean nidos u ocupan nidos de otros organismos, ovipositan y se encargan de la alimentación de las larvas (GOULSON, 2010b). Al emerger los primeros adultos, por lo regular hembras, estas inician su papel como obreras y ayudan a la alimentación de las larvas restantes, por un periodo de 3 a 4 semanas, sin embargo finalizado este periodo la hembra reina muere y las descendientes quedan libres para formar su propio nido. En este sentido se reduce la alimentación en el mismo y finalmente es abandonado (GOULSON, 2010b). Lo anterior explica no solo la reducción de polen en el mes mayo y junio, también el abandono de los nidos.

Por otra parte, al detallar la composición, riqueza, diversidad y dominancia al interior de las especies fueron notorias ciertas diferencias (Figura 1A, 1B y 2A). En el mes de abril B. pullatus esta compuesto principalmente por Calceolaria, Euphorbia, Miconia, Phyllanthus, entre otros; mientras que en $B$. atratus granos que no están registrados en $B$. pullatus aparecen con frecuencia como: Acanthaceae, Vallea,
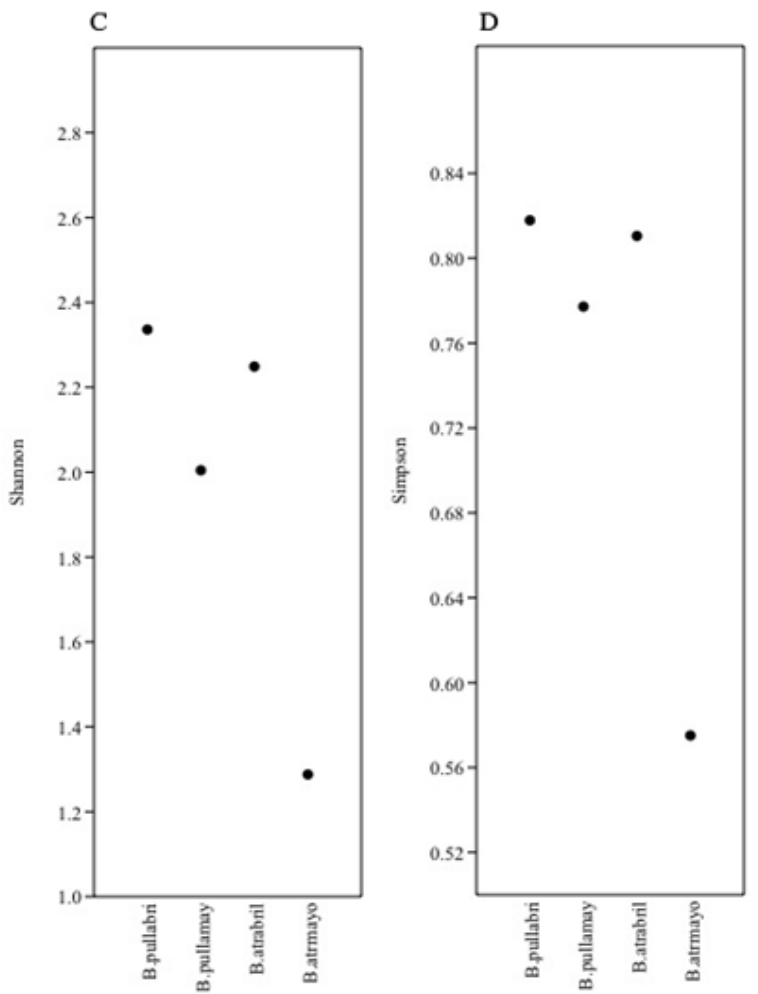

Figura 2. A, Número de tipos polínicos por cada especie en los meses de muestreo. B, resultados del índice de Margalef por cada especie en los meses de muestreo. C, resultados del índice de Shannon por cada especie en los meses de muestreo. D, resultados del índice de Simpson por cada especie en los meses de muestreo. 
Croton, Stigmaphyllon, Sida y Piper. En el caso de la composición en el mes de mayo, si se observan grandes variaciones en ambas especie. En B. pullatus, Clibadium, Gynoxys, Phyllanthus, Borreria, Cestrum y Solanum son importantes; mientras que en $B$. atratus solo Acmella, Clibadium y Solanum. En el caso de la diversidad (Shannon) y dominancia (Simpson) los resultados son similares a los observados en la riqueza y número de taxones (Figura 2A, 2B, 2C y 2D); en este sentido, $B$. pullatus es el grupo más rico y diverso, mientras que $B$. atratus se observa menor diversidad producto de la baja riqueza observada en el mes de mayo.

Estos resultados muestran el papel que juegan estas especies en los bosques subandinos como prestadores de servicios ecosistémicos. Aunque hallar granos de polen en las heces de estas dos especies, no sugiere necesariamente un proceso de polinización, si se espera que a aquellos taxones cuyo porcentaje de granos es alto (Fabaceae, Asteraceae, entre otros) exista una gran posibilidad de eventos de polinización en los bosques subandinos (KREMEN et al., 2007).

Asimismo la gran abundancia de granos de polen de Solanaceae, Fabaceae y Asteraceae principalmente, permiten sugerir dos aspectos. El primero, que durante los meses de muestreo existió mayor disponibilidad e incluso cercanía de estas especies a los nidos y por lo tanto de sus recursos florales. El segundo punto radica en la preferencia de Bombus hacia estos clados, lo cual ha sido demostrado para otras especies en los Andes colombianos (RUBIO-FERNÁNDEZ, 2012); no obstante, la carencia de información sobre la proporción de polen generada por la flora y análisis de las visitas de los abejorros, solo permite sugerir la preferencia, sin poder determinar tal selección con mayor precisión.

De igual forma, la alta proporción de los granos de granos de polen tipo Solanum sp1 y sp2, pueden ser explicado por la presencia de cultivos de Lulo (Solanum quitoense Lam.) en las cercanías a los nidos. Algunos estudios en zonas andinas ya han reportado el uso de B. atratus (Almanza 2007) y B. terrestris (MESSINGER et al., 2016) como polinizadores importantes de este cultivo, sin embargo en Pamplonita este recurso no ha sido establecido. Bajo este contexto, se llevaron a cabo comparaciones morfológicas y morfométricas entre los granos hallados en el presente trabajo con descripciones realizadas en granos de polen de $S$. quitoense (MERCADO-GÓMEZ et al., 2015), hallado grandes similitudes que asocia a los granos de Solanum sp1 a S. quitoense. Estos datos confirman el uso potencial de especies de Bombus nativas como prestadores de servicios ecosistemicos a especies de uso agrícola.

En conclusión, el análisis palinológico en muestras de heces sobre especies del genero Bombus, permite establecer un listado de especies de angiospermas que posiblemente hacen parte de la dieta de estas especies, la cual está asociada a los periodos de nidifación, ya que los análisis fueron realizados directamente de muestras recolectadas en nidos con presencia de larvas (observaciones realizadas en campo). El número elevado de granos de polen de taxones como Fabaceae, Asteraceae, Solanaceae, sugiere la importancia de las especies del genero Bombus como un prestador de servicios ecosistémicos en los bosques andinos, tanto de especies nativas de estos bosques, como de grupos de uso comercial.

\section{Referencias}

BARTH, O.M. 2013. Palynology serving the stingless bees. Págs. 285-294 en P. Vit; M. Pedro; D. Roubik (eds.). Pot-Honey: A legacy of stingless bees. Springer New York.

BOGOTÁ, R.G. 2002. El polen de la subclase Asteridae en el páramo de Monserrate. Manizales: Publ co. Manizales, Colombia.

BOGOTÁ, R.G.; LAMPREA, S.J.; RANGEL, J.O. 1996. Atlas palinológico de la clase Magnoliopsida en el páramo Monserrate. Págs. 131-191 en Fundación-ecosistemas-Andino-ECOAN (ed.). El páramo sistema a proteger. CODICE Ltda, Bogotá.

CASTELLANOS-POTENCIANO, B.P.; RAMÍREZ-ARRIAGA, E.; ZALDIVAR-CRUZ, J.M. 2012. Análisis del contenido polínico de mieles producidas por Apis mellifera L. (Hymenoptera: Apidae) en el estado de Tabasco, México. Acta zoológica mexicana 28:13-36.

ERDTMAN, G. 1952. Pollen morphology and plant taxonomy Angiosperms. Alquimist and Winksell. Stockholm. 
ESTAY, P.; WAGNER, V.A.; ESCAFF, G.M. 2001. Evaluación de Bombus dahlbomii (Guér.) como agente polinizador de flores de tomate (Lycopersicon esculentum (Mill)), bajo condiciones de invernadero. Agricultura Técnica 61:113-119.

GOULSON, D. 2010a. Bumblebees behaviour, ecology and conservation. Oxford University Press. Oxford.

GOULSON, D. 2010b. Social organization and conflict. Págs. 21 - 43 en D. Goulson (ed.). Bumblebees behaviour, ecology and conservation. Oxford University Press. Oxford.

HOFFMANN, W.; TORRES, A.; NEUMANNC, P. 2004. A scientific note on the nest and colony development of the Neotropical bumble bee Bombus (Robustobombus) melaleucus. Apidologie 35:449-450.

KREMEN, C.; WILLIAMS, N.M.; BUGG, R.L.; FAY, J.P.; THORP, R.W. 2004. The area requirements of an ecosystem service: crop pollination by native bee communities in California. Ecology Letters 7 (11):1109-1119.

MERCADO-GÓMEZ, J. 2007. Polen de la clase Magnoliopsida para el Volcán- Pamplona (Norte de Santander Colombia). Trabajo de Pregrado. Departamento de Biología y Química, Universidad de Pamplona, Colombia.

MERCADO-GÓMEZ, J.; JIMÉNEZ-BULLA, L.C.; SÁNCHEZ-MONTAÑO, L.R. 2011. Polen de las Magnoliopsida en el Volcán -Pamplona (Colombia) I: familias Apiaceae, Asteraceae, Cunoniaceae, Ericaceae, Fabaceae y Gentianaceae. Caldasia 33 (2):619-635.

MERCADO-GÓMEZ, J.D.; CARMONA-DUQUE, D.T.; JIMÉNEZ-BULLA, L.C.; ACEITUNO-BOCANEGRA, F.J. 2015. Flora palinológica de la cuenca media del río San Eugenio, Risaralda (Cordillera Central), Colombia. Actualidades Biológicas 37 (103):185-200.

MESSINGER, J.; MARTINI, M.M.F.; ROSSI, G.; SAMUELS, J.; LAUERER, M. 2016. Successful pollination of the Neotropical crop Solanum quitoense by Bombus terrestris: behaviour, efficiency and yield. Journal of Applied Entomology 140 (1-2):124-134.

NATES-PARRA, G.; MONTOYA, P.; CHAMORRO, F.; RAMÍREZ, N.; GIRALDO, C.; OBREGÓN, D. 2013. Origen geográfico y botánico de mieles de Apis mellifera (Apidae) en cuatro departamentos de Colombia. Acta biológica colombiana $18(3): 427-438$.

NATES-PARRA, G.; RODRÍGUEZ, Á. 2011. Forrajeo en colonias de Melipona eburnea (Hymenoptera: Apidae) en el piedemonte llanero (Meta, Colombia). Revista Colombiana de Entomología 37:121-127.

OBREGÓN, D.; RODRÍGUEZ, A.; CHAMORRO, F.J.; NATES-PARRA, G. 2013. Botanical origin of pot honey from Tetragonisca angustula Latreille in Colombia. Págs. 337-346. en P. Vit; S.R.M. Pedro; D.W. Roubik (ed.). Pot-Honey: A Legacy of Stingless Bees. Springer Verlag. Berlin, Germany.

PUNT, W.; HOEN, P.P.; BLACKMORE, S.; NILSSON, S.; THOMAS, A.L. 2007. Glossary of pollen and spore terminology. Review of Palaeobotany and Palynology 143:1-81.

RAMÍREZ-ARRIAGA, E.; MARTíNEZ-BERNAL, A.; RAMÍREZ-MALDONADO, N.; MARTÍNEZ-HERNÁNDEZ, E. 2016. Análisis palinológico de mieles y cargas de polen de Apis mellifera (Apidae) de la región Centro y Norte del estado de Guerrero, México. Botanical Science 94 (1):141-156.

ROUBICK, D.W.; MORENO, J.E. 1991. Pollen and spores of Barro Colorado island. Monographas in systematic botany. Miss. Bot. Gard 36:1-268.

RUBIO-FERNÁNDEZ, D. 2012. Disponibilidad, uso y preferencia por los recursos florales en una comunidad de abejorros (Hymenoptera: Apidae: Bombus) en el páramo de Chingaza. Facultad de Ciencias, Departamento de Biología, Universidad Nacional de Colombia.

SCHIESTL, F.P.; JOHNSON, S.D. 2013. Pollinator-mediated evolution of floral signals. Trends in Ecology \& Evolution 28 (5):307-315. 
TELLERIA, M.C. 1998. Palynological analysis of food reserves found in a nest of Bombus atratus (Hym. Apidae). Grana 37 (2):125-127.

TEPER, D. 2006. Food plants of Bombus terrestris L. as determined by pollen analysis of feces. Journal of Apicultural Science 50 (2):101-108.

VEGA-SUÁREZ, L.M. 2009. Iniciación y cría de Bombus atratus (Hymenoptera: Apidae): un aporte a la conservación. Departamento de Biología y Química Universidad de Pamplona. 